\title{
INSCRIPTIONS SUR LES BOUCLIERS CIRCULAIRES DE L'ORIENT MUSULMAN ${ }^{1}$
}

\author{
PAR \\ LUDVIK KALUS
}

NOUS avons présenté dans deux numéros précédents de cette revue deux études concernant les boucliers circulaires musulmans, dont une suivait l'évolution et le développement des éléments caractéristiques de ces boucliers en s'éfforçant de définir des groupes de boucliers typiques pour une période ou un milieu ${ }^{2}$, tandis que l'autre observait les aspects technique et morphologique de ces objets ${ }^{3}$. Les deux articles sont les résultats d'une étude approfondie d'une centaine de boucliers que nous avons étudiés dans plusieurs musées d'Europe ${ }^{4}$. Pendant nos recherches, nous avons aussi rélevé systématiquement les inscriptions se trouvant sur ces boucliers et dans cet article, nous présentons les inscriptions que nous avons trouvées dans la «population» examinée, en les classant d'après leur contenu.

Cet «inventaire» des inscriptions se trouvant sur les boucliers circulaires de l'Orient musulman est loin d'être exhaustif, car il est le résultat de recherches effectuées sur une «population» limitée. Néanmoins, il peut donner un aperçu sur la nature des inscriptions sur ce type de boucliers et présente en plus quelques inscriptions qui n'ont pas été jusqu'à maintenant publiées, dont certaines sont très caractéristiques pour ce genre d'objets, comme nous avons pu le constater au cours de visites dans d'autres musées, visites postérieures à nos recherches systématiques dans ce domaine.

1 Cet article constitue une partie de notre thèse de doctorat de $3^{\mathrm{c}}$ cycle, présentée en 1974 à l'Université de Paris-Sorbonne (Paris IV).

2 Ludvik Kalus, Boucliers circulaires de l'Orient musulman (Évolution et utilisation), dans «Gladius», XII, 1974, pp. 59-133, 30 fig.

3 ID., Aspects morphologique et technique des boucliers circulaires de l'Orient musulman, dans «Gladius», XIII, 1977, pp. 25-61.

${ }^{4}$ Les musées visités sont les suivants: Historisches Museum de Berne (Suisse), Musée de l'Armée de Paris, Musée de l'Homme de Paris (France), Kunsthistorisches Museum de Vienne, Historisches Museum der Stadt Wien, Heeresgeschichtliches Museum de Vienne (Autriche), Náprstkovo Museum de Prague (Tchécoslovaquie). Nous tenons à exprimer une fois de plus nos remerciements aux directions respectives de ces musées de nous avoir autorisé d'étudier les objets se trouvant dans leurs collections. 


\section{FRÉQUENCE DES INSCRIPTIONS SUR LES BOUCLIERS}

La plupart des inscriptions que nous avons relevées se trouvent sur les boucliers en métal, où les deux tiers des boucliers examinés sont pourvus d'une ou de plusieurs inscriptions. Sur les boucliers en peau les inscriptions sont rares, seulement deux boucliers sur les vingt-cinq que nous avons vus en portent ${ }^{5}$. Sur les boucliers en «osier», nous avons trouvé trois boucliers avec des inscriptions ${ }^{6}$, sur une «population» de douze boucliers. L'unique bouclier en papier mâché étudié est sans inscription ${ }^{\text {? }}$.

\section{TECHNIQUES D'EXÉCUTION DES INSCRIPTIONS}

Elles correspondent en principe aux techniques employées pour exécuter le décor sur un bouclier donné. Sur les boucliers en métal c'est la ciselure, mais les caractères peuvent encore être soulignés par l'incrustation, souvent superficielle, d'or ou d'argent, ou bien les inscriptions peuvent être exécutées à l'eau forte, technique fréquente notamment en Iran à partir du Xviı ${ }^{\mathrm{e}}$ siècle. Souvent les inscriptions sont seulement incrustées, sur un fond plat, et dans un seul cas elles sont écrites au pinceau ${ }^{8}$. La même technique du pinceau est employée pour les inscriptions sur les boucliers en peau ${ }^{9}$. Sur les boucliers en "osier» tressé, les inscriptions sur les flancs sont en fils multicolores tressés, sur le disque central en métal, elles sont incrustées d'or.

\section{RÉPARTITION DES INSCRIPTIONS SUR LA BASE DES BOUCLIERS}

Les inscriptions se trouvent exclusivement sur la surface externe de la base des boucliers. Ici, leur emplacement par excellence est la bordure formée d'une ou de plusieurs bandes circulaires. Cette bande

5 Berne, Musée d'Histoire, no. 969 (Inde de l'Ouest, lère moitié du xix ${ }^{c}$ siècle); Vienne, Historisches Museum der Stadt Wien, no. 126.229 (Turquie, Zème moitié du Xvile siècle).

6 Paris, Musće de l'Armée, no. I 87 (Iran, xvie ou xvir siècle); Paris, Musée de l'Armée, no. I 90 (Turquie, xvI ${ }^{\mathrm{e}}$ ou xvII ${ }^{\mathrm{e}}$ siècle); Vienne, Heeresgeschichtliches Museum, l'exposition permanente (Turquie, $\mathrm{xvII}^{\mathrm{e}}$ siècle).

7 Berne, Musée d'Histoire, no. 1285 (Inde du nord-ouest, fin du xix ${ }^{\mathrm{e}}$ siècle).

8 Berne, Musée d'Histoire, no. 1480 (Iran, xix ${ }^{\mathrm{e}}$ siècle).

9 Berne, Musée d'Histoire, no. 969 (Inde de l'ouest, lère moitié du XIX ${ }^{\mathrm{C}}$ siècle); Vienne, Historisches Museum der Stadt Wien, no. 126.229 (Turquie, 2ème moitié du xvII ${ }^{e}$ siècle). 
peut être sans aucune division interne et l'inscription est alors sans interruption ${ }^{10}$. Mais le plus souvent les inscriptions sont réparties dans les cartouches, soit dans chaque cartouche d'une bande ${ }^{11}$, soit en alternance avec les cartouches contenant un décor floral ou figuratif ${ }^{12}$. Si la bande de la bordure est partagée par deux types de cartouches en alternance, alors chaque type de cartouches contient une inscription différente et les parties d'une inscription alternent avec les parties d'une autre ${ }^{13}$. On trouve aussi des bordures formées de deux bandes dont une bande contient une inscription continue et l'autre bande contient une autre inscription partagée dans les cartouches ${ }^{14}$. Une bande circulaire autour de l'umbo du bouclier peut aussi servir d'encadrement à une inscription ${ }^{15}$, surtout s'il s'agit d'un bouclier en «osier» tressé où une telle bande forme en principe la bordure du disque central en métal ${ }^{16}$. Rares sont les inscriptions dans les médaillons répartis dans le champ du bouclier ${ }^{17}$.

L'un des boucliers étudiés ${ }^{18}$ peut être considéıé comme bouclier «à inscriptions», car trois inscriptions différentes sont en effet réparties

10 Par exemple Prague, Musće Naprstek, no. 4506 (Iran, XIXe siècle); Paris, Musée de l'Armée, no. G 739 (Iran, xix e siècle); Paris, Musée de l'Armée, no. I 6670 (Iran, XIX ${ }^{\mathrm{e}}$ siècle).

11 Par exemple Prague, Musée Naprstek, no. 4504 (Iran, 2ème moitié du XIx ${ }^{\mathrm{e}}$ siècle); Prague, Musée Naprstek, no. 4507 (Iran, 2ème moitić du xix ${ }^{\mathrm{e}}$ siècle); Berne, Musée d'Histoire, no. 904 (Iran, 2ème moitié du XvIII ${ }^{\mathrm{e}}$ siècle); Berne, Musée d'Histoire, no. 1480 (Iran, XIX ${ }^{*}$ siècle).

12 Par exemple Prague, Musée Naprstek, no. A 2680 (Iran, fin du $x^{2} I I^{e}$ ou début du $\mathrm{XIX}^{\mathrm{e}}$ siècle); Prague, Musée Naprstek, no. 19479 (Iran, fin du XviII $^{\mathrm{e}}$ ou début du XIXe siècle); Prague, Musée Naprstek, no. 4453 (Iran, XIXe siècle); Prague, Musée Naprstek, no. 43604 (Iran, fin du XviII ou début du XIXe siècle); Prague, Musée Naprstek, no. A 2681 (Iran, fin du xvIII ${ }^{c}$ ou début du $\mathrm{XIX}^{\mathrm{c}}$ siècle); Berne, Musée d'Histoire, no. 931 (Iran, lère moitié du xix ${ }^{e}$ siècle); Berne, Musée d'Histoire, no. 1426 (Inde du Nord-Ouest, fin du XIXe ou début du $\mathrm{Xx}^{\mathrm{e}}$ siècle); Paris, Musée de l'Armée, no. 2852 (Iran, 1ère moitié du XIX ${ }^{\mathfrak{c}}$ siècle).

${ }_{13}$ Par exemple Prague, Musée Naprstek, no. 4495 (Iran, fin du xvIII ${ }^{\mathrm{e}}$ ou début du XIX siècle); Berne, Musée d'Histoire, no. Ps. 39 (Iran, xIXe siècle).

14 Par exemple Prague, Musée Naprstek, no. 4505 (Iran, xIx ${ }^{e}$ siècle); Prague, Musée Naprstek, no. 19478 (Iran, XIX" siècle).

15 Par exemple Vienne, Kunsthistorisches Museum, no. C 94 (Moyen-Orient arabe (?), XVII ${ }^{\mathrm{c}}$ ou $\mathrm{xvIII}^{\mathrm{e}}$ siècle); Vienne, Historisches Museum der Stadt Wien, no. 126.229 (Turquie, 2ème moitié du Xvir siècle). siècle).

16 Par exemple Paris, Musée de l'Armée, no. I 90 (Turquie, $\mathrm{Xv1}^{\mathrm{c}}$ ou $\mathrm{XvII}^{\mathrm{c}}$

17 Berne, Musée d'Histoire, no. 939 (Iran, 2ème moitié du XviII ${ }^{\mathrm{e}}$ ou XIx ${ }^{\mathrm{e}}$ siècle); Paris, Musée de l'Homme, no. 62.71 .29 '(Iran, lère moitié du XIX ${ }^{\mathrm{e}}$ siècle); Berne, Musée d'Histoire, no. 969 (Inde de l'Ouest, 1ère moitié du xix ${ }^{\mathrm{e}}$ siècle); Paris, Musée de l'Armée, no. I 87 (Iran, $\mathrm{xvI}^{\mathrm{e}}$ ou $\mathrm{xvII}^{\mathrm{e}}$ siècle).

I8 Vienne, Kunsthistorisches Museum, no. C 85 (Turquie, moitié du Xvi siècle). 
dans la bande circulaire autour de l'umbo, dans la bande circulaire formant la bordure ainsi que dans les sept médaillons répartis régulièrement dans le champ.

\section{DIVISION DES INSCRIPTIONS D'APRÈS LEUR NATURE}

Les inscriptions se trouvant sur les boucliers sont en langue arabe ou persane. Nous les présentons en les groupant selon leur nature.

\section{INSCRIPTIONS À CARACTÈRE RELIGIEUX}

Un certain nombre d'inscriptions mentionne le nom de Dieu, qui peut être accompagné d'un des Noms divins. Ainsi nous trouvons mentioné, à plusieurs reprises, sur les flancs d'un bouclier en «osier» tressé ${ }^{19}$ le mot au, «Dieu», réparti dans huit groupes dont chacun contient le mot Dieu cinq fois. Ailleurs ${ }^{20}$ on trouve répétée, dans sept

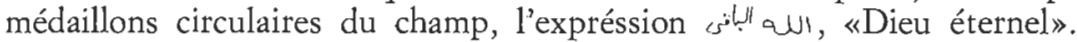
Sur l'umbo d'un autre bouclier ${ }^{21}$, nous remarquons l'expression $3-$ i «Dieu est la vérité», ainsi que quatre lettres $\rho \rightarrow 1$ qui forment le mot $\overrightarrow{1}$, , «ois clément». L'expression $2 \mu_{\text {; }} \mathrm{ll}$, , «uissance à Dieu», est répetée dix-neuf fois dans une bande autour de l'umbo d'un autre bouclier ${ }^{2}$.

On ne remarque pas souvent des inscriptions contenant des textes coraniques. On trouve le verset 13 de la sourate LXI, une fois très

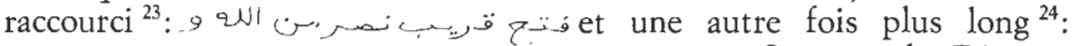

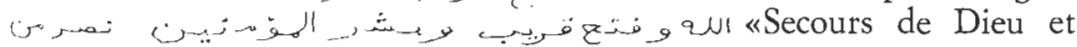

19 Vienne, Heeresgeschichtliches Museum, l'exposition permanente (Turquie, xvII ${ }^{\mathrm{e}}$ siècle).

20 Vienne, Kunsthistorisches Museum, no. C 85 (Turquie, moitié du $\mathrm{xvI}^{\mathrm{e}}$ siècle). Les inscriptions se trouvant sur ce bouclier ont été publiées dans Eduard Freih. von SACKen, Die K. K. Ambraser-Sammlung, t. I, Vienne, 1855, pp. 208209.

21 Vienne, Historisches Museum der Stadt Wien, no. 126.229 (Turquie, 2ème moitié du xvII $^{*}$ siècle). Les inscriptions se trouvant sur ce bouclier ainsi que sa description ont été publiées dans Johan SzENDREI, Ungarische kriegsgeschichtliche Denkmäler in der Milleniums-Landes-Ausstellung, Budapest, 1896, p. 606, no. de cat. 3250 .

${ }^{22}$ Vienne, Kunsthistorisches Museum, no. C 94 (Moyen-Orient arabe, Xvil ${ }^{\mathrm{c}}$ ou XVIII siècle).

${ }_{23}$ Berne, Musée d'Histoire, no. 969 (Inde de l'Ouest, lère moitié du XIX siècle).

24 Voir la note 21. 
prochain succès! Annonce la bonne nouvelle aux Croyants! " ${ }^{25}$. A la fin de cette deuxième citation est ajoutée l'invocation cor l «Ô Muhammad!». Sur un bouclier ${ }^{26}$, on trouve encore un autre verset coranique, qui est le verset 1 de la sourate XLVIII, mais sans la basmala:

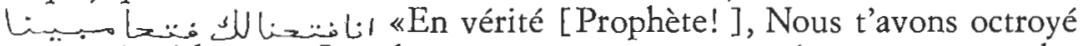
un succès éclatant». Les deux versets se trouvent très souvent sur des drapeaux et des étendards et sur d'autres armes musulmanes, surtout de l'époque ottomane ${ }^{27}$.

Nous pouvons considérer que l'inscription contenant les noms des quatre premiers califes a aussi dans une certaine mesure un caractère religieux ${ }^{28}$. Les noms de ces quatre califes sont répartis autour de l'umbo de l'un de nos boucliers ${ }^{29}$ de la façon suivante:

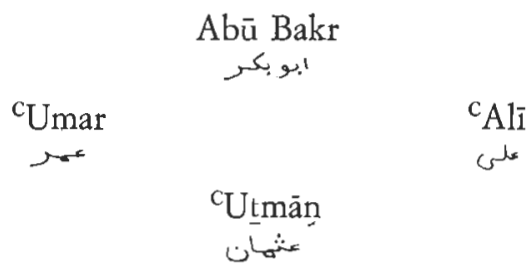

Un quatrain arabe contenant l'invocation à ${ }^{\mathrm{C}} \mathrm{Ali}$, trouvé sur un

25 Un autre bouclier avec le verset 13 de la sourate LXI a été publié dans Georges de KaEmmerer, Arsenal de Tsarskoé-Sélo, ou Collection d'armes de Sa Majesté l'Empereur de toutes les Russies, St. Pétersbourg, 1869, pl. XVII/2. Le verset 13 de la sourate LXI est accompagné sur ce bouclier des versets 256, 257,258 et 259 de la sourate II. siècle).

26 Berne, Musée d'Histoire, no. 969 (Inde de l'Ouest, lère moitié du XIX ${ }^{\mathrm{e}}$

${ }^{27}$ Cf. par exemple Z. Zygulski jr., Turkish Trophies in Poland and the Imperial Ottoman Style, tiré à part de Armi Antiche, Bolletino dell'Accademia di S. Marciano, Torino, numero speciale per il $6^{\circ}$ Congresso dell'Associazione Internazionale dei Musei d'Armi e di Storia Militare, Zurigo, 15-20 Maggio 1972, p. 30; Korán, traduction tchèque d'Ivan HrbeK, Prague, 1972, p. 751 verš 13 et p. 768 verše $1-4$.

${ }_{28}$ Les quatre premiers califes, Abū Bakr, 'Umar, 'Utmān et ${ }^{c} A l \bar{i}$, sont appelés al-hulafā̄ al-rāšidūn, «les califes qui suivent le droit chemin» ou «les califes orthodoxes». Ils font partie des Dix élus (al-ašara al-mubaššara), parmi lesquels ils occupent les quatre premières places. Les Dix élus sont tous des Compagnons du Prophète. A propos des Dix élus voir Henri Laoust, La profession de foi d'Ibn Batta (Traditionniste et jurisconsulte musulman d'école banbalite, mort en Irak à cúubarâ en 387/997), Damas, 1958, pp. 113-117; Dominique SourdeL, A propos des «Dix élus», dans REI, XXXI, 1963, pp. 111-114.

29 Vienne, Historisches Museum der Stadt Wien, no. 126.229 (Turquie, 2ème moitié du XVII ${ }^{\mathrm{e}}$ siècle). Voir note 21. 
bouclier ${ }^{30}$, peut témoigner de la tendance shî̀ite, qui est un peu affaiblie par la mention de Muhammad dans le dernier hémistiche ${ }^{31}$ :

(entièrement effacé)

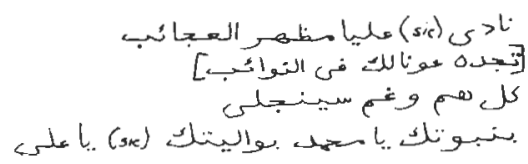

«Invoque ${ }^{\mathrm{C}} \mathrm{Al}$ ī qui fait paraître les prodiges

Tu le trouveras secourable dans le malheur

Toute angoisse, tout chagrin se dissiperont

Par ta qualité de Phophète, ô Muhammad, par ta qualité

d'Ami de Dieu, ô ${ }^{\mathrm{C}} \mathrm{Ali} \gg{ }^{32}$.

Une longue inscription, répartie dans huit cartouches ${ }^{33}$, paraît contenir quelques sentences religieuses, mais son exécution est très inexacte et il ne nous a pas été possible de la déchiffrer intégralement Voici ce que nous avons été en mesure de déchiffrer:

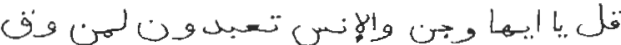

$$
\begin{aligned}
& \text { all }
\end{aligned}
$$

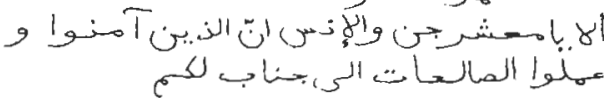

30 Paris, Musée de l'Armée, no. I 90 (Turquie, $x^{2} I^{\mathrm{e}}$ oul xvII ${ }^{\mathrm{e}}$ siècle).

31 On trouve souvent ce quatrain sans aucune mention de Muhammad. Nous

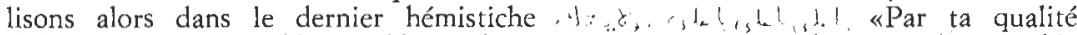
d'Ami de Dieu, ô 'Alī, ô "Alī, $\hat{o}$ 'Alī», et cette omission du nom du Prophète témoigne d'une tendance shî'ite. D'après Reinaud, les sunnites utilisaient aussi ce quatrain, mais ce sont justement eux qui ajoutaient aux deux derniers ou seulement au dernier hémistiche quelques mots se rapportant à Muhammad, ce qui est le cas sur le bouclier étudié par nous. Voir M. Reinaud, Monuments arabes, persans et turcs du cabinet de M. le Duc de Blacas et d'autres cabinets, Paris, 1828, t. II, pp. 168-172. Ce quatrain est mentionné chez Reinaun dans la liste des inscriptions fréquemment utilisées sur les armes. On le trouve également souvent sur les talismans musulmans, cf. par exemple A. Chabouillet, Description des antiquités el objets d'art composant le cabinet de M. Louis Fould, Paris, 1861, pp. 177-78; Etienne ComBe, Notes d'arcbéologie musulmane, III. Trois amulettes, dans BIFAO, XV, 1918, p. 207, no. 1 .

32 Le même quatrain se trouve sur un bouclier publié dans E. REHATSEK, Orientalische Rüstungstïcke, dans $Z D M G$, XXXVI, 1882, pp. 656-7, où la lecture diffère un peu de la notre et de celle présentée par REINAUD. Mais cette inscription n'était pas réservée seulement aux armes, comme en témoigne par exemple un encrier de l'époque safavide, publié dans A. S. Melikinn-Chinvani, Le bronze iranien, coll. Musée des Arts Décoratits, 1, Paris, 1973, p. 100. La traduction donnée par Melikian-Chinvani nous a servi d'exemple pour notre traduction.

33 Berne, Musée d'Histoire, no. 1480 (Iran, XIX ${ }^{\mathrm{e}}$ siècle). 


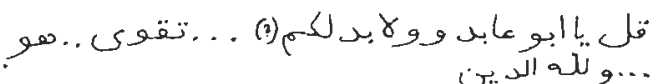

$$
\begin{aligned}
& \text { و }
\end{aligned}
$$

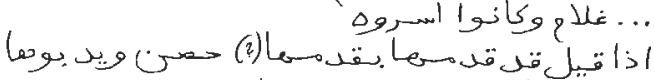

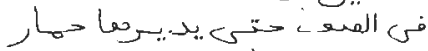

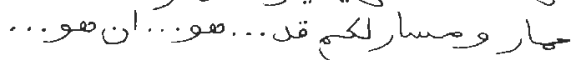

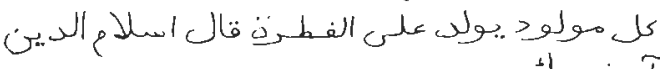

$$
\begin{aligned}
& \text { th } \sin 1
\end{aligned}
$$

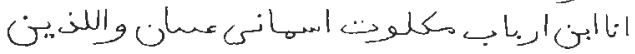

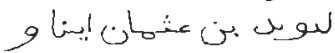

La lecture de cette inscription n'étant pas sûre et complète, nous ne donnons pas ici sa traduction. Notons néanmoins que nous avons lu avec sûreté le début de l'inscription dans la ligne no. 7 , où nous avons retrouvé une citation tirée du hadì ${ }^{34}$.

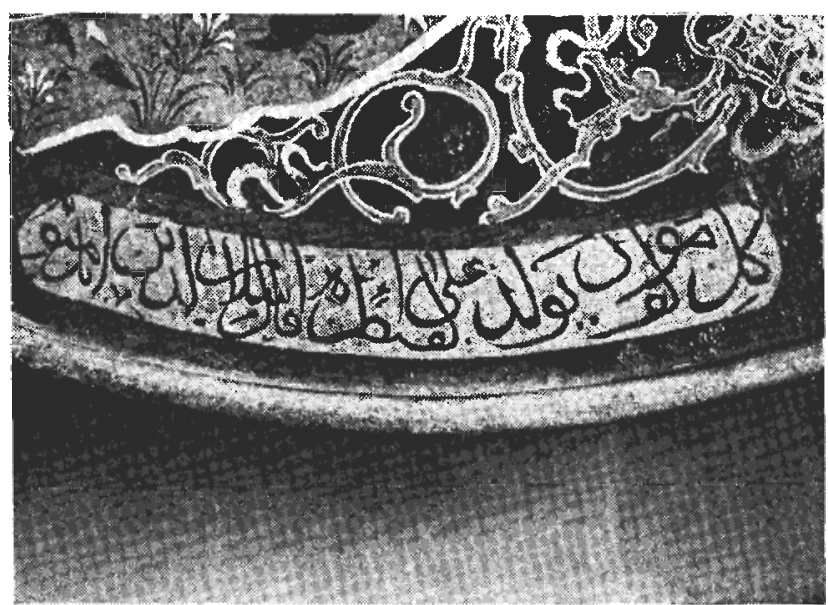

FIG. 1.-Iran, XIXe siècle (Berne, Musée d'Histoire, no. 1480) (Cliché: Kalus)

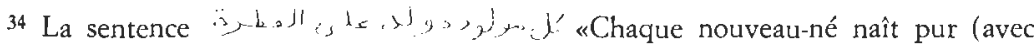
un sentiment religieux inné dans le coeur) » se trouve, d'après J. Brugman, Concordance et indices de la tradition musulmane, t. V, Leyde, 1965, p. 180, dans les

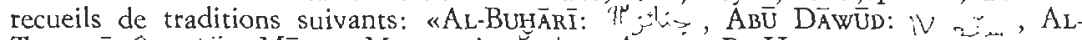

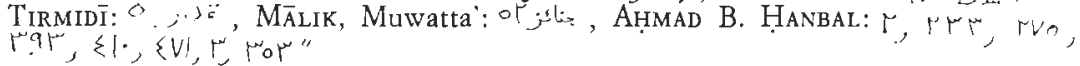


La présence de ces inscriptions religieuses, sauf la dernière qui se trouve sur un bouclier qui est un objet typique «de curiosité», avait pour but d'attirer la protection divine sur le propriétaire du bouclier.

\section{INSCRIP'TIONS EXPRIMANT DES VOEUX}

Une autre catégorie d'inscriptions est celle exprimant des voeux en faveur du propriétaire et de l'utilisateur du bouclier. Elles ne sont pas nombreuses, en réalité seulement deux boucliers examinés portent des inscriptions de ce genre.

Le premier exemple est celui d'une inscription répartie dans les sept cartouches d'une bordure ${ }^{35}$ :

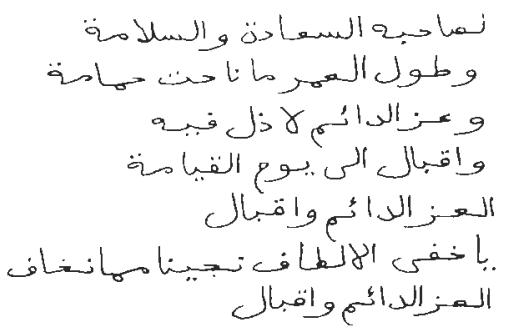

(1) «A son propriétaire bonheur et salut

(2) Et durée de la vie tant que la colombe roucoule

(3) Et puissance éternelle sans déshonneur

(4) Et prospérité jusqu'au jour du jugement dernier

(5) Puissance éternelle et prospérité

(6) Ô toi qui cache (en toi) les bontés, sauve nous de ce que nous craignons

(7) Puissance éternelle et prospérité»

Autour de l'umbo du même bouclier on trouve encore:

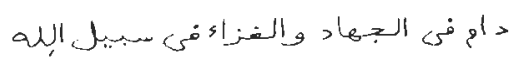

«Qu'il continue dans la guerre et dans le combat pour la religion sur le chemin de Dieu! »

Sur les champs d'un bouclier en «osier» ${ }^{36}$ nous trouvons répété dans trois cartouches > béni»).

35 Vienne, Kunsthistorisches Museum, no. C 85 . Cf. note 20.

36 Paris, Musée de l'Armée, no. I 87 (Iran, $\mathrm{XVI}^{\mathrm{e}}$ ou $\mathrm{xvII}^{\mathrm{e}}$ siècle). 


\section{INSCRIPTION CONTENANT LES TITRES D'UN SOUVERAIN}

Ce groupe est assez vaste, néanmoins l'authenticité historique de ces inscriptions est très contestable, au moins en ce qui concerne les boucliers que nous avons examinés. En réalité, dans très peu de cas seulement le nom du souverain est mentionné et presque toujours les mêmes titres se répètent sans règle logique, étant souvent accompagnés de caractères ou de groupes de caractères n'ayant aucun sens. On peut dire que la plupart de ces inscriptions se trouve déjà presque à la limite de la pseudo-épigraphie, ayant surtout un but décoratif. Une autre raison de leur présence sur les boucliers est de les faire passer pour plus anciens. Ici, nous sommes en présence d'objets exécutés en Iran au $\mathrm{XIX}^{\mathrm{e}}$ ou au début du $\mathrm{XX}^{\mathrm{e}}$ siècles, destinés surtout aux étrangers.

Les inscriptions les plus complètes sont ici les inscriptions sur deux boucliers ${ }^{37}$, où elles occupent sans interruption la bande unique formant la bordure. Ces deux inscriptions contienent le nom d'un shāh safavide, pour l'une ${ }^{38}$ c'est le nom du shāh Sulaymān, sans doute le premier (1666-1694), car Sulaymān II est habituellement mentionnée comme Sulaymān «tānī» (au moins sur les monnaies), et il n'a régné que quelques mois de la fin de l'année 1749 au début de l'année 1750.

La deuxième inscription ${ }^{39}$ mentionne le nom du shāh ${ }^{\mathrm{C}} \mathrm{Abbās}$ II (tānī) qui a régné entre 1642-1666. Mais sur le même bouclier se trouve la date [1] 115 qui correspond aux années 1703-1704 de l'ère chrétienne et il est presque évident que cette date et l'inscription ont été inscrites en même temps, mais beaucoup plus tardivement, au $\mathrm{XIX}^{\mathrm{e}}$ siècle, d'après le caractère du décor.

Ceci est aussi valable pour le bouclier qui porte le nom du shāh Sulaymān et ces inscriptions n'ont par conséquent aucune valeur historique. Il est très difficile de trouver le commencement de ces deux textes car les titres de ces deux shäh se suivent, étant seulement interrompus par le nom du shāh.

Le texte de l'inscription contenant le nom du shāh ${ }^{\mathrm{C}}$ Abbās II (16421666) est le suivant:

37 Prague, Musée Naprstek, no. 4506 (Iran, XIx ${ }^{\mathrm{e}}$ siècle); Paris, Musée de l'Armée, no. G 739 (Iran, xix ${ }^{\mathfrak{e}}$ siècle).

33 Prague, Musée Naprstek, no. 4506 (Iran, XIx siècle).

39 Paris, Musée de l'Atmée, no. G 739 (Iran, xix siècle). 


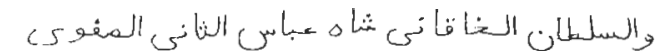

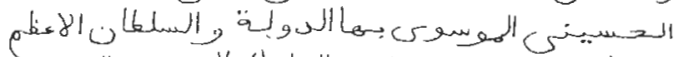

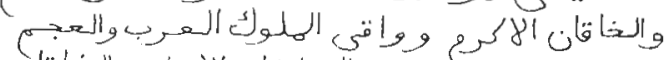

$$
\begin{aligned}
& \text { 光 }
\end{aligned}
$$

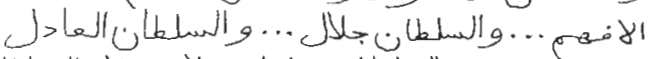

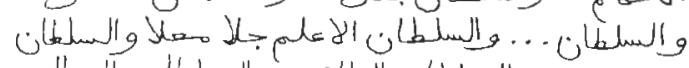

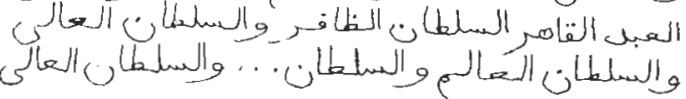

$$
\begin{aligned}
& \text { - juturlog }
\end{aligned}
$$

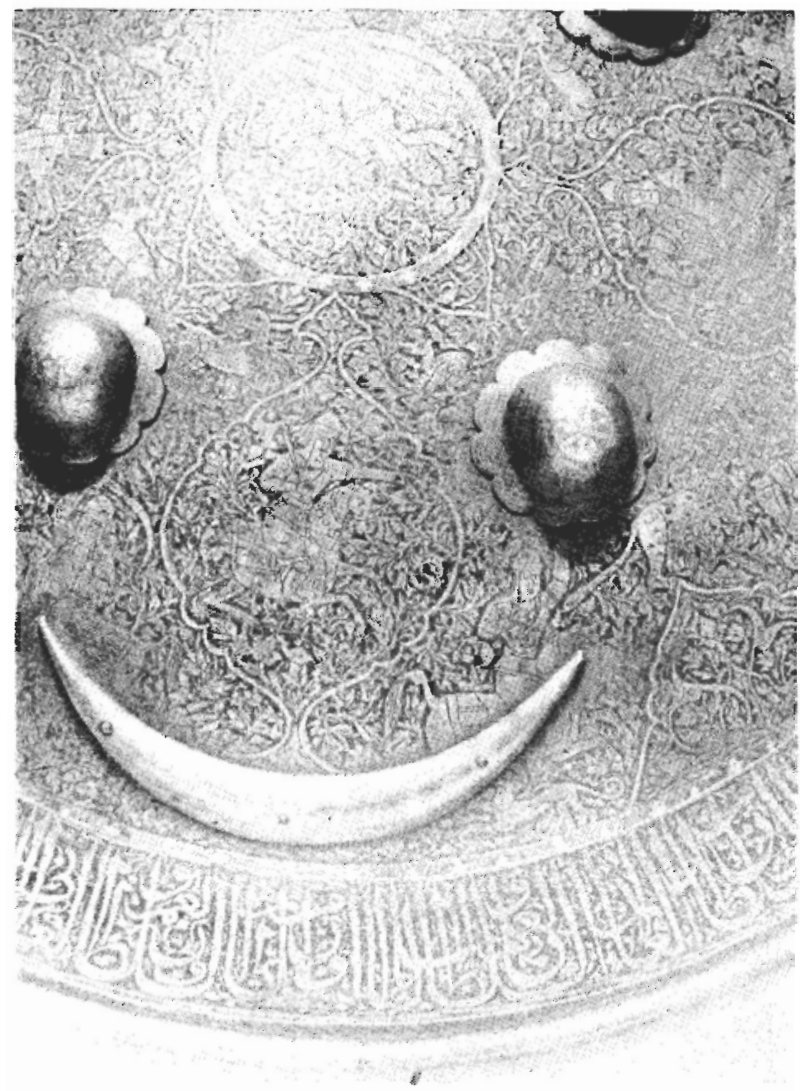

Fig. 2.-Iran, $X I X^{e}$ siècle (Paris, Musée de l'Armée, no. G 739) (Cliché: Service Photographique du Musée de l'Armée) 
«Le sultan impérial, shāh ${ }^{\mathrm{c}}$ Abbās II, Safavide, qui se réclame de Husayn, qui se réclame de Mūsā, la beauté de l'empire, le sultan très grand, l'empereur très noble, le protecteur des rois des Arabes et des non-Arabes, le sultan très noble, le sultan très grand, le souverain très intelligent... le sultan, grandeur..., le sultan juste, le sultan..., le sultan très savant, splendeur de la grandeur, le sultan serviteur et vainqueur, le sultan victorieux, le sultan élevé, le sultan savant, le sultan..., le sultan élevé, le sultan...»

Nous ne sommes pas en mesure de donner le texte entier de l'inscription contenant le nom du shāh Sulaymān (1666-1694), car la qualité de la photographie ne nous le permet pas. Nous nous contentons de présenter seulement la partie où le nom du shāh est mentionné:

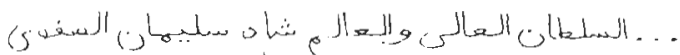

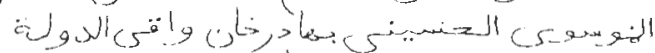

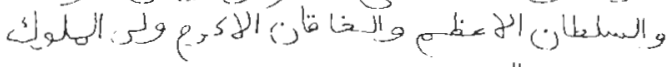

$$
\begin{aligned}
& \cdots e=1
\end{aligned}
$$

«... le sultan élevé et savant, shāh Sulaymān, Safavide, qui se réclame de Mūsā, qui se réclame de Husayn, le hān (souverain) courageux, le protecteur de l'empire, le sultan très grand, l'empereur très noble, le tuteur des rois des Arabes et des non-Arabes...»

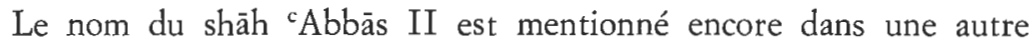
inscription, assez confuse, que nous ne pouvons lire que partiellement. Elle se trouve dans les huit cartouches de la bordure du bouclier qui fut sans doute fabriqué postérieurement à l'époque du règne du shāh ${ }^{\mathrm{c}}$ Abbās II. Nous avons déchiffré dans cette inscription les fragments suivants ${ }^{40}$ :

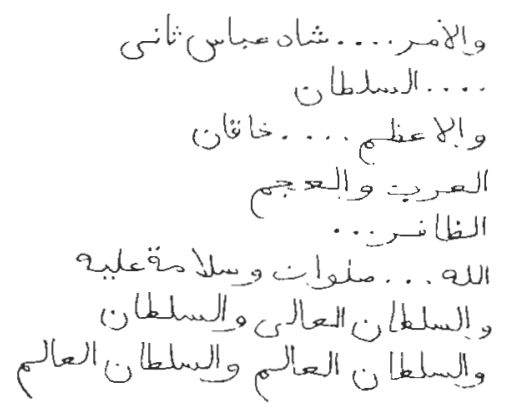

40 Prague, Musée Naprstek, no. 19478 (Iran, xrxe siècle). 
(1) «L'ordre... shāh ' Abbās II

(2) ..... le sultan

(3) et le très grand... l'Empereur

(4) des Arabes et des non-Arabes...

(5) le victorieux...

(6) Dieu... que les bénédictions et la salutation soient sur lui

(7) le sultan élevé et le sultan

(8) le sultan savant et le sultan savant»

Deux inscriptions d'un caractère assez semblable se trouvent réparties dans les huit médaillons circulaires de la bordure de deux boucliers ${ }^{41}$, où ces médaillons alternent avec des cartouches contenant des vers persans, les mêmes sur les deux boucliers. Une de ces inscriptions mentionne le nom de Nādir Shāh, l'autre ne contient pas le nom d'un personnage concret.

Voici le texte de l'inscription mentionnant le nom de Nādir Shāh ${ }^{42}$ :

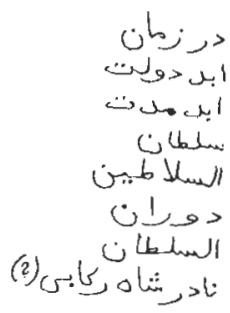

«(1) Du temps (2) de l'éternellement puissant (régnant) (3) de l'éternellement durant (4) le sultan (5) des sultans (6) de l'époque (7) le sultan (8) Nädir Shāh ( + un mot)» ${ }^{43}$.

Le texte de l'autre inscription est le suivant ${ }^{44}$ :

41 Prague, Musée Naprstek, no. 4495 (Iran, fin du xvirI ${ }^{\mathrm{e}}$ ou début du XIX ${ }^{\mathrm{e}}$ siècle); Berne, Musée d'Histoire, no. Ps. 39 (Iran, XIX ${ }^{\mathrm{e}}$ siècle).

42 Prague, Musée Naprstek, no. 4495 (Iran, fin du XvIII ${ }^{e}$ ou début du XIx siècle).

${ }_{43}$ Après le nom de Nādir Shāh il y a un mot que nous lisons rokābi $\left(r, 5, L_{1}\right)$, ce qui peut signifier d'après F. Steingass, $A$ Comprebensive Persian-English Dictionnary, Beyrouth, 1970, p. 584: «A small dish, plate, or saucer; a cup, a gublet; a cup-bearer; a led horse for parade; a sword carried on a horse's side; one who holds the stirrup, that his master may mount; a kind of rupee current in Lucknow; a kind of Damascus oil carried on camels». Mais nous ne voyons aucune signification pour ce mot dans le contexte donné, après le nom de Nādir Shāh.

44 Berne, Musée d'Histoire, no. Ps. 39 (Iran, xix ciècle). 


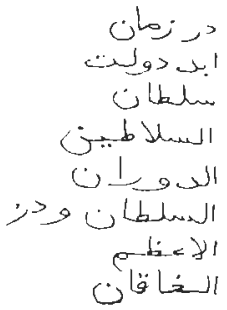

«(1) Du temps (2) de l'éternellement puissant (régnant) (3) le sultan (4) des sultans (5) de l'époque (6) le sultaa (trois lettres en plus) (7) très grand (8) l'Empereur».

Il y a encore trois autres boucliers où nous trouvons des inscriptions contenant le mot sultan accompagné de divers titres, sans mentionner de nom de souverain ${ }^{45}$ (Fig. 8). Mais ce sont toujours les mêmes titres qui se répètent et ces inscriptions n'ont aucune valeur historique.

\section{VERS PERSANS ${ }^{46}$}

Ce groupe d'inscriptions est le plus fréquent sur les boucliers examinés. Certains de ces vers, dont plusieurs contiennent le mot «separ» qui signifie boulier, se répètent sur plusieurs objets. On compte en tout sept morceaux différents de poésie.

Dans quatre cartouches de la bordure de deux boucliers sont répartis deux vers du Šāhnāme de Firdousī. Ces vers sont les mêmes mais ils diffèrent néanmoins dans le premier hémistiche. Le mètre de ces vers est motaqāreb. Voici le texte d'une de ces deux inscriptions ${ }^{47}$ :

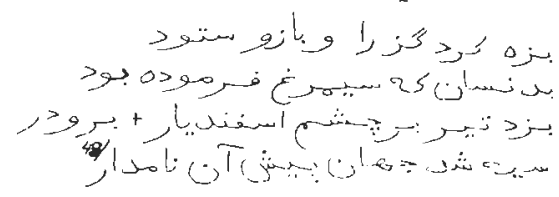

45 Prague, Musée Naprstek, no. 4505 (Iran, XIX e siècle); Prague, Musée Naprstek, no. 4453 (Iran, XIx ${ }^{c}$ siècle); Prague, Musée Naprstek, no. 4507 (Iran, 2ème moitié du $\mathrm{XIX}^{\mathrm{e}}$ siècle).

46 Nous remercions Mr. C. H. DE Fouchecour, professeur à l'Institut national des langues et civilisations orientales, qui a eu la gentillesse de revoir avec nous la lecture ainsi que la traduction française des inscriptions persanes se trouvant sur les boucliers étudiés.

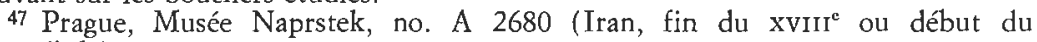
XIXe siècle).

48 Voir Toannes Augustus Vullers, Firdusii liber regum qui inscribitur

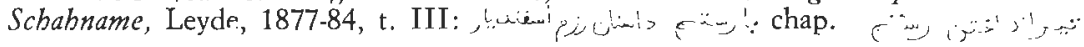


«(1) Il ${ }^{49}$ a mis la flèche dans la corde et il a fait l'éloge de la force

(2) Ainsi que Sĩmorg l'a ordonné

(3) Il a tiré la flèche dans l'oeil d'Esfandyār

(4) Le monde s'est noirci devant cet illustre (héros)».

Le texte ainsi que la répartition des vers dans les cartouches sur l'autre bouclier ${ }^{50}$ sont un peu différents:

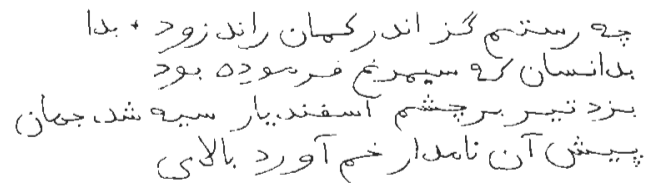

(1) «Quand Rostam a mit rapidement la flèche dans l'arc

(2) Comme Sīmorg l'a ordonné

(3) Il a tiré la flèche dans l'oeil d'Esfandyār, Le monde s'est noirci

(4) devant cet illustre (héros). Il a courbé le sommet» ${ }^{51}$.

Sur quatre boucliers ${ }^{52}$, dont même deux fois sur l'un ${ }^{53}$, se répètent des vers bien connus du Golestān de $\mathrm{Sa}^{\mathrm{C}} \mathrm{di}^{\mathrm{i}}$. Ils sont répartis soit dans les cartouches d'une bande de la bordure, soit dans les médaillons placés dans le champ. Le texte et la traduction sont les suivants:

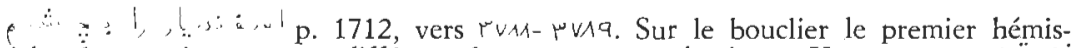
tiche du premier vers est différent, le texte comme le donne Vullers est ,$\cdots ;, i, 1, i, i, 5, j, 1,3$. Dans le deuxième hémistiche du premier vers Vullers donne, l, il , sur le bouclier nous trouvons

49 Il 's'agit de Rostam, l'un des héros du S̄āhnāme.

50 Prague, Musée Naprstek, no. A 2681 (Iran, fin du xvilite ou début du $\mathrm{XIX}^{\mathrm{e}}$ siècle).

51 Voir Joannes Augustus Vullers, Firdusii liber regum qui inscribitur

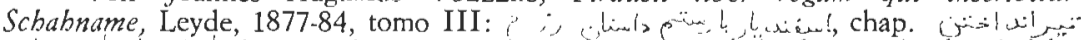
t. du vers rvq: Sur le bouclier le début du premier hémistiche du premier vers est

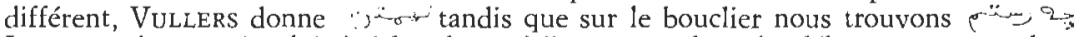
Le texte du premier hémistiche du troisième vers dont le début se trouve dans le cartouche no. 4 est d'après Vullers is $s_{-\infty}, j, \geq$, français «Il a courbé le sommet du cyprès droit».

52 Berne, Musée d'Histoire, no. 939 (Iran, 2ème moitié du xviII ${ }^{\mathrm{e}}$ ou xix $\mathrm{x}^{\mathrm{e}}$ siècle); Berne, Musée d'Histoire, sans no. (Iran, 2ème moitié du xviII ${ }^{\circ}$ ou 1ère moitié du $\mathrm{XlX}^{\mathrm{e}}$ siècle); Paris, Musée de l'Armée, no. 2852 (Iran, lère moitié du XIX siècle); Paris, Musée de l'Homme, no. 62.71 .29 (Iran, lère moitié du XIX siècle). siècle).

53 Berne, Musée d'Histoire, no. 939 (Iran, 2ème moitié du xvilI 


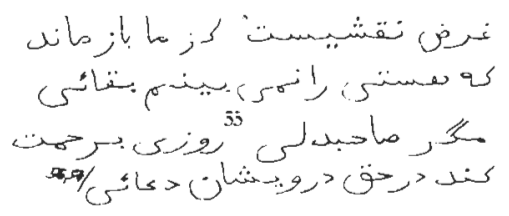

(1) Le but de ce décor est qu'il nous survive

(2) Car je ne vois pas de stabilité dans l'existence

FIG 3.-Iran, lère moitié du XIX siècle (Paris, Musée de l'Homme, número 62.71.29) (Cliché: Lem-

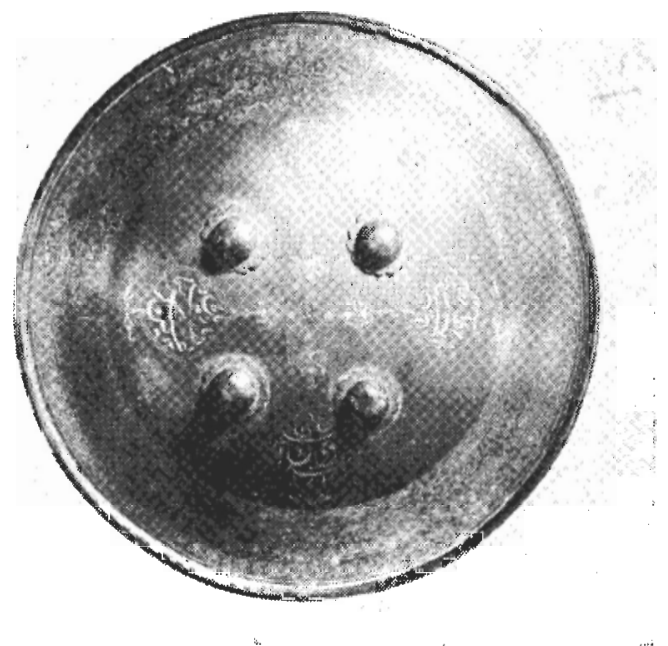

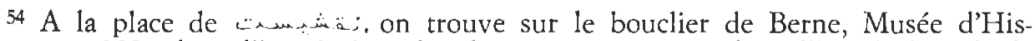
toire, no. 939, dans l'inscription du champ dans l'inscription de la bordure..... sur le bouclier de Berne, Musée d'Histoire, s.n, on trouve

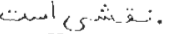

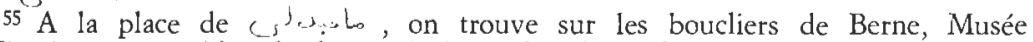
d'Histoire, no. 939 (dans le champ ainsi que dans la bordure) et s.n. inscrit

56 Dans la bordure du bouclier de Berne, Musée d'Histoire, no. 939, Te dernier hémistiche n'est pas complet: à la place du mot (e- nous trouvons su qui n'a aucun sens et le dernier mot s a n'est pas complet non plus, nous ne trouvons que $>$.

57 Voir SADI , Golestān, Kritičeskij text, perevod, predislovije i primečanija P. M. Alieva, Moscou, 1959, .... Lis; (Introduction), p. 29 (texte persan) et note 23 . 
(3) Peut-être un jour, par miséricorde, un homme pieux

(4) Va-t-il faire une prière pour les derviches» ${ }^{58}$.

Un do-beyt ${ }^{59}$ persan, qui fait la louange du bouclier, se trouve dans la bordure de trois boucliers ${ }^{60}$. Comme les trois textes de ces vers diffèrent dans quelques détails, nous les reproduisons ici séparément pour chaque bouclier.

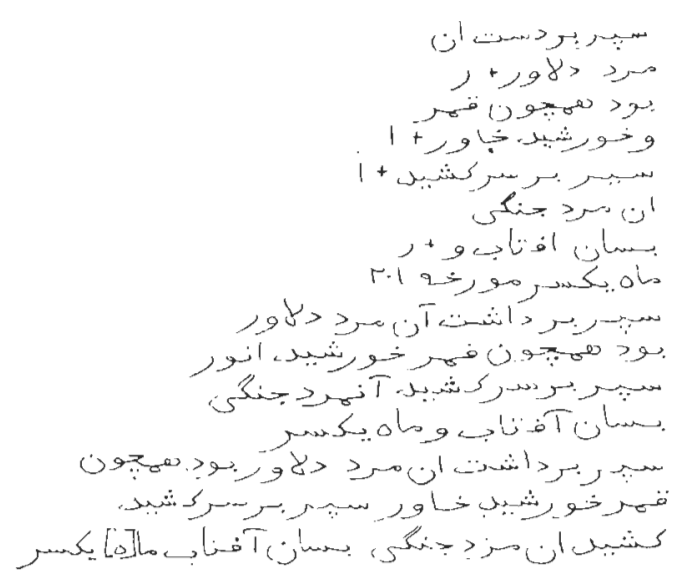

58 D'après T. N. MukharJI, Art manufactures of India (Abridged edition), Calcutta, 1888, pp. $87-88$, on peut encore souvent trouver sur les boucliers d'autres vers du Golestān de $S A^{\complement} \bar{D} \bar{l}$. Il s'agit des vers suivants:

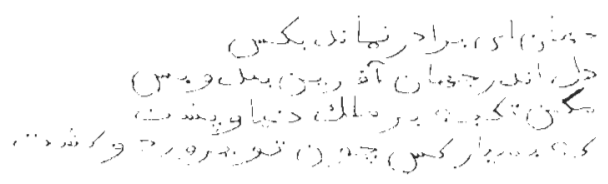

(voir SAcDī, Golestān, Kritičeskij text, perevod, predislovije i primečanija P. M. Alieva, Moscou, 1959, chap. I: ...... ; , . . , , p. 33 (texte persan).

59 Do-beytī est une forme poétique persane comprenant deux vers, la rime est faite d'après le système «aaba».

60 Prague, Musée Naprstek, ло. 19479 (Iran, fin du xviII ${ }^{\mathrm{e}}$ ou début du $\mathrm{xIx}^{\mathrm{e}}$ siècle); Berne, Musée d'Histoire, no. 931 (Iran, 1ère moitié du xix ${ }^{e}$ siècle); Berne, Musée d'Histoire, s.n. (Iran, 2ème moitié du $\mathrm{XVIII}^{e}$ ou 1ère moitié du XIx siècle).

${ }_{61}$ Prague, Musée Naprstek, no. 19479 (Iran, fin du XviII ${ }^{\mathrm{e}}$ ou début du XIx siècle).

${ }_{62}$ Berne, Musée d'Histoire, no. 931 (Iran, 1ère moitié du XIX ${ }^{\mathrm{C}}$ siècle).

63 Berne, Musće d'Histoire, s.n. (Iran, 2ème moitié du xviII ou lère moitié du XIX ${ }^{e}$ siècle). 


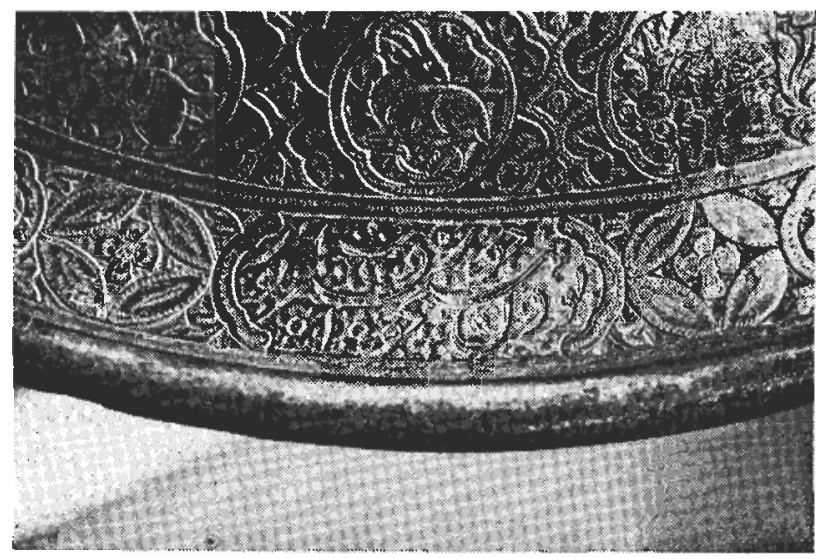

FIg. 4--Iran, 1ère moitié du XIX siècle (Berne, Musée d'Histoire, no. 931) (Cliché: Kalus)

A-(1-2) «Le bouclier à la main de cet homme courageux

(3-4) Etait comme la lune (et) le soleil levant

(5-6) Ce combattant se défend

(7-8) Comme le soleil et la lune ensemble; daté [1]201» ${ }^{64}$

B - (1) «Cet homme courageux a saisi le bouclier

(2) Il était comme la lune (et) le soleil très brillant

(3) Ce combattant se défend

(4) Comme le soleil et la lune ensemble

C - (1) Cet homme courageux a saisi le bouclier, Il était comme

(2) la lune (et) le soleil levant, Se défend

(3) ce combattant, Comme le soleil et la lune ensemble» ${ }^{65}$.

Une quit ${ }^{c} \mathrm{e}^{66}$ composée de trois vers persans, accompagnée d'un fard ${ }^{67}$, contenant l'éloge des qualités du bouclier, sont répartis dans

64 L'année 1201 de l'hégire correspond à l'année 1786-87 de l'ère chrétienne.

65 En ce qui concerne le dernier hémistiche de ce do-beytī, nous avons vu au Museo, Stibbert de Florence un bouclier où on lisait assez clairement

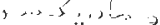

to $\mathrm{Qitị}^{\mathrm{a}} \mathrm{a}$ est une forme poétique persane dont la rime est faite d'après le système «aa, ba, ca» etc. et dont le sujet a habituellement un caractère philosophique ou éthique et peut exprimer une idéee ou une réflexion.

67 Dans la poétique persane, le fard est un vers isolé. Les rimes des deux hémistiches sont faites d'après le système «aa» ou «ab». 
huit cartouches de la bordure de trois boucliers ${ }^{68}$. Sur un quatrième bouclier ${ }^{69}$ on trouve encore le premier vers de cette $\mathrm{qit}^{\mathrm{C}} \mathrm{e}$ accompagné du même fard, auxquels est ajouté encore un vers dont nous donnons le texte plus loin. Sur l'un des boucliers examinés ${ }^{70}$, l'exécution de cette inscription est assez fruste: souvent des caractères manquent ou bien ne son exécutés qu'approximativement. Il s'agit sans doute de l'oeuvre d'un artisan qui copiait un modèle sans comprendre le contenu. C'est surtout l'inscription sur un bouclier de Berne (Musée d'Histoire, s.n.) qui nous sert de guide pour le déchiffrement, hélas, une petite partie en a été effacée par la rouille. Mais en comparant les endroits moins clairs avec les deux autres inscriptions, nous pouvons être en mesure de donner comme exemple le texte approximatif de ce bouclier:

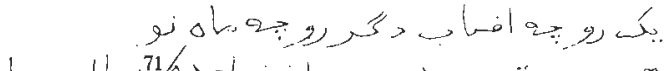

$$
\begin{aligned}
& \text { l, } \\
& 1,1, g^{1+01}, \text { ho } 0 \text {, }
\end{aligned}
$$

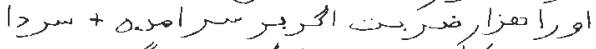

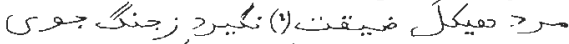

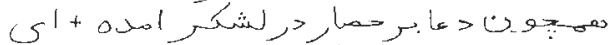

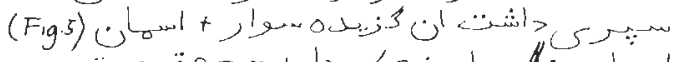

$$
\begin{aligned}
& \text { رo } 0,0,0 \text { a }
\end{aligned}
$$

(1) «Une face ressemblait au soleil et l'autre à la nouvelle lune

(2) On aurait dit la pleine lune près de l'astre

(3) Compagnon solide... parmi les combattants

(4) S'il reçoit mille coups

(5) L'homme fort (?) n'est pas gêné par celui qui cherche la guerre

(6) Il devient au milieu de l'armée comme la bénédiction sur la forteresse

(7) Cet excellent cavalier a un bouclier

(8) Qui a la couleur du ciel et l'effet d'un miroir»

68 Prague, Musée Naprstek, no. 4495 (Iran, fin du xviII ${ }^{\mathrm{e}}$ ou début du XIX ${ }^{\mathrm{C}}$ siècle); Berne, Musée d'Histoire, s.n. (Iran, 2ème moitié du XviII ${ }^{e}$ on 1ère moitié du XIX ${ }^{\mathrm{e}}$ siècle); Berne, Musée d'Histoire, no. Ps. 39 (Iran, XIX siècle).

${ }^{69}$ Prague, Musée Naprstek, no. 43604 (Iran, fin du xv1II ${ }^{\circ}$ ou début du $X I X^{e}$ siècle).

70 Berne, Musée d'Histoire, no. Ps. 39 (Iran, xıxe siècle).

71 On remarque ces deux premiers hémistiches encore sur un bouclier persan daté du XviI ${ }^{\mathrm{e}}$ siècle, qui se trouve au Museo Poldi Pezzoli à Milan. Sur ce même bouclier se trouvent aussi les vers du Golestān de SAcDī «Le but de ce décor est qu'il nous survive...», dont nous avons parlé infra, note 57 et le texte corresponde רt. Voir Heinrich Gluck et Enst Díez, Die Kunst des Islam, Berlin, 1925, p. 586 pl. 458. 


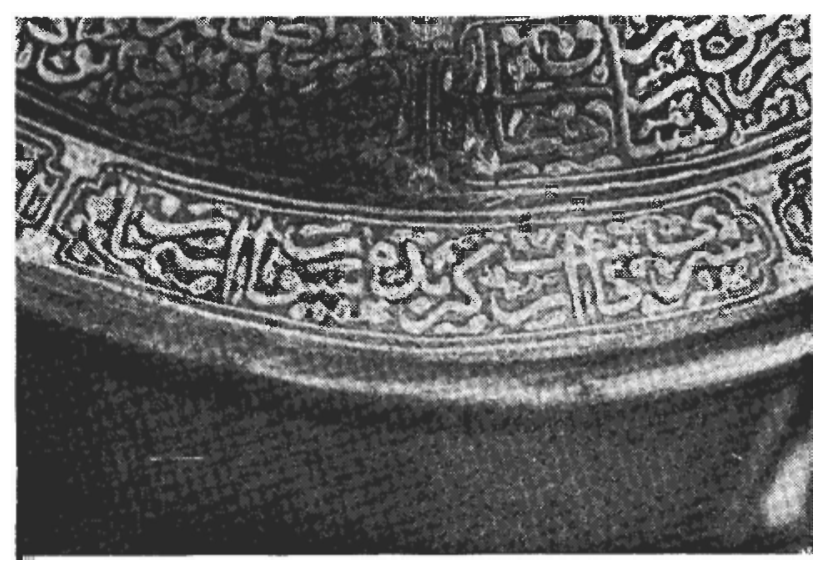

FIG. 5.-Iran, 2ème moitié du XVIII $I^{\mathrm{e}}$ ou lère moitié du XIX ${ }^{e}$ siècle (Berne, Musée d'Histoire, s.n.) (Cliché: Kalus)

Un autre fard fait également mention du bouclier. Nous le trouvons sur trois boucliers ${ }^{72}$, toujours accompagné d'autres vers. Voici son texte:

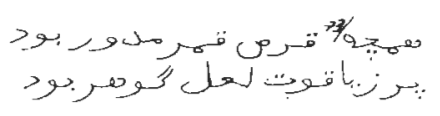

(1) «Il était rond comme le disque de la lune

(2) Il était plein de rubis et de pierres précieuses»

Ce vers se trouve sur un bouclier à côté des vers du Golestān de $\mathrm{Sa}^{\mathrm{c}} \mathrm{d} \bar{\imath}^{74}$, et sur un autre bouclier ${ }^{75}$ à côté des vers du Golestān de $\mathrm{Sa}^{\mathrm{C}} \mathrm{di}$ et des vers précédents ainsi qu'à côté des vers qui commencent par "Cet homme courageux a saisi le bouclier...» que nous avons données ci-dessus. Sur un troisième bouclier ${ }^{76}$ on trouve ce vers

72 Prague, Musée Naprstek, no. 43604 (Iran, fin du xvIII ${ }^{\mathrm{e}}$ ou début du XIx siècle); Berne, Musée d'Histoire, s.n. (Iran, 2ème moitié du xviII ${ }^{\circ}$ ou lère moitié du xix $\mathrm{x}^{\mathrm{e}}$ siècle); Paris, Musée de l'Hlomme, no. 62.71 .29 (Iran, 1ère moitié du $\mathrm{XIX}^{\mathrm{e}}$ siècle).

${ }^{73}$ Sur le bouclier de Paris, Musée de l'Homme, no. 62.71 .29 on trouve à la place de ? e le mot 0 ?

${ }^{74}$ Paris, Musée de l'Homme, no. 62.71 .29 (Iran, 1ère moitié du XIX ${ }^{\mathrm{c}}$ siècle).

75 Berne, Musée d'Histoire, s.n. (Iran 2ème moitié du XvIII ${ }^{e}$ ou lère moitié du XIX $X^{\circ}$ siècle).

${ }_{76}$ Prague, Musée Naprstek, no. 43604 (Iran, fin du Xvill ${ }^{\mathrm{e}}$ ou début du XIX siècle). 
accompagné du premier vers du morceau précédent ainsi que du vers isolé qui est ajouté à la fin de ce morceau. Cette inscription est répartie dans six cartouches:

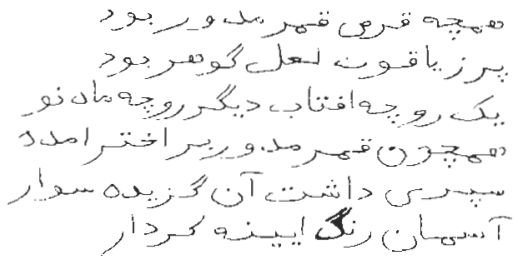

(1) «Il était rond comme le disque de la lune

(2) Il était plein de rubis et de pierres précieuses

(3) Une face ressemblait au soleil et l'autre à la lune

(4) On aurait dit la pleine lune près de l'astre

(5) Cet excellent cavalier a un bouclier

(6) Qui a la couleur du ciel et l'effet d'un miroir»

Un autre do-beytī se trouve dans quatre cartouches d'un autre bouclier ${ }^{77}$. Ces cartouches sont répartis dans la bande extérieure de la bordure, tandis que dans la bande intérieure de cette bordure on remarque dans quatre cartouches les vers du Golestān de $S^{c}{ }^{c} \mathrm{~d} \overline{1}$ mentionnés ci-dessus. L'exécution de cette inscription n'est pas excellente et à défaut d'un matériel de comparaison - cette inscription ne se trouve que sur un bouclier- nous ne sommes pas en mesure d'en donner le texte exact. Nous y distinguons ce qui suit:

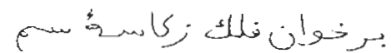

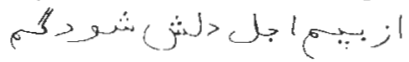

$$
\begin{aligned}
& \text { C. }
\end{aligned}
$$

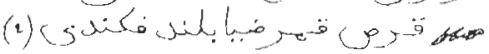

(1) «Sur la table du ciel, de la coupe de...(?)

(2) Son coeur est éperdu d'une grande peur

(3) Sur ta cuisse (se trouve) le signe du shāh de l'univers

(4) Le disque de la lune...» (Fig. 6).

Un morceau de poésie comprenant quatre vers et faisant l'éloge d'un casque, est réparti dans les huit cartouches de la bande intérieure de la bordure d'un des boucliers étudiés ${ }^{78}$. Son texte est le suivan ${ }^{79}$ (Fig. 7):

77 Paris, Musée de l'Armée, no. 2852 (Iran, 1ère moitié du xix siècle).

78 Berne, Musée d'Histoire, no. 904 (Iran, 2ème moitié du xviII ${ }^{\mathfrak{2}}$ siècle).

$79 \mathrm{La}$ traduction allemande de cette inscription a été publiée, pas tout à fait correctement, dans Rudolf ZELLER et Ernest F. ROHRER, Orientaliscbe Sammlung 


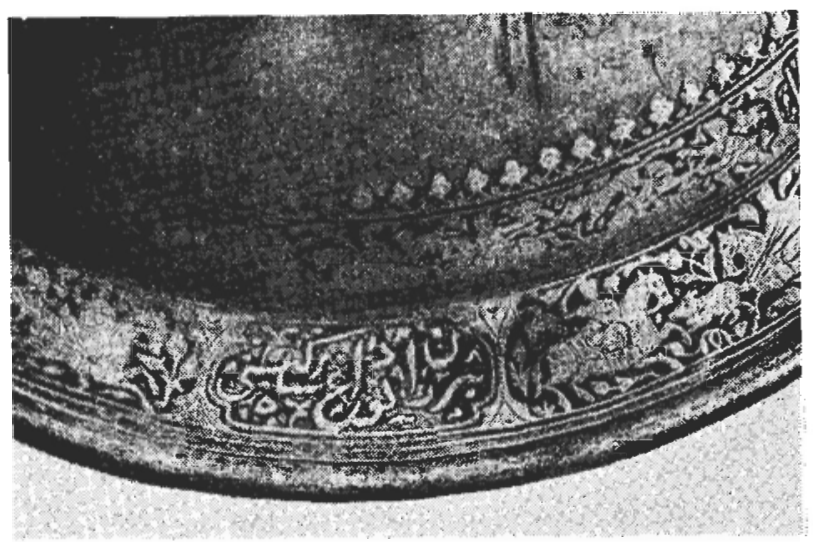

FIG, 6.-Iran, lère moitié du XIX siècle (Paris, Musée de l'Armée, no. 2852) (Cliché: Kalus)

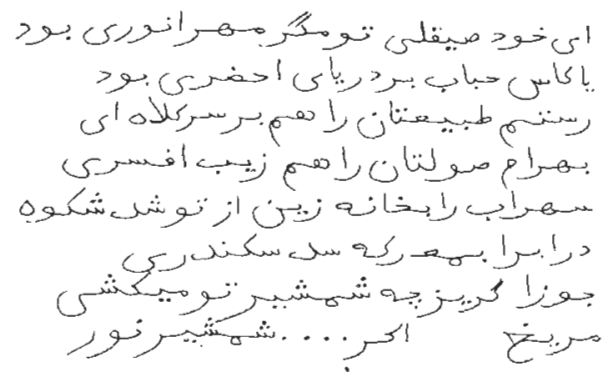

(1) «Ô casque brillant, tu est peut-être le soleil très brillant

(2) Ou tue es une coupe de bulle sur l'océan vert

(3) Tu es la coiffure sur la tête des gens au caractère de Rostari

(4) Pour ceux qui ont la bravoure de Bahrām tu es l'ornement du diadème

(5) Grâce à toi Sohrāb a reçu la grandeur en selle

(6) Tu es pour Darius dans la bataille le barrage d'Eskander

Henri Moser-Cbarlottenfels; Bescbreibender Katalog der Waffensammlung, Berne, 1955 , p. 85, accompagnée d'une description du bouclier en question. On trouve la même inscription sur un casque qui est également dans la collection Moser au Musée d'Histoire de Berne et dont la traduction allemande a été également publice dans Ibid., p. 48-49. 


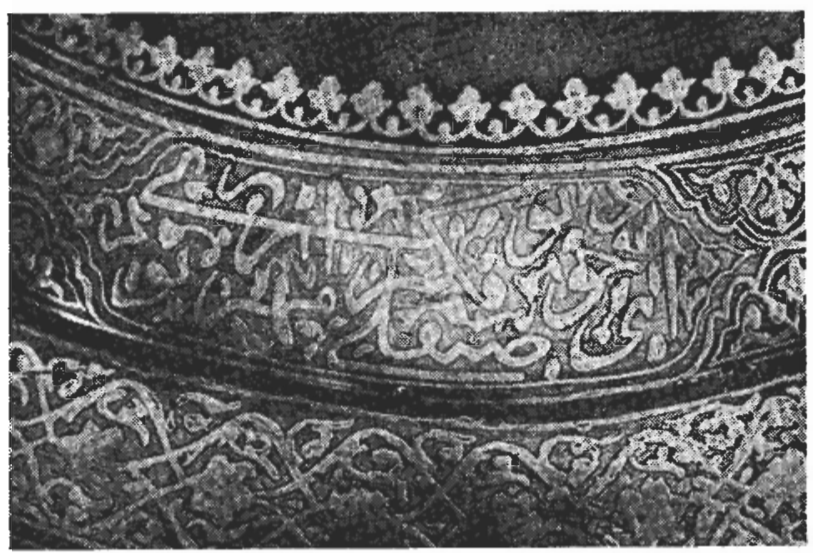

FIG. 7.-Iran, 2ème moitié du XVIII" siècle (Berne, Muséc d'Histoire, no. 904) (Cliché: Kalus)

(7) Les Gémaux (ou l'Orion) s'enfuient quand tu dégaines ton épée

(8) Mars... l'épée de lumière»

Les lacunes dans certains vers persans ici présentés ne sont pas impossibles à combler, car il existe sans doute un grand nombre de boucliers contenant ces inscriptions et les endroits peu clairs ou mal exécutés sur un bouclier pourront être lus en les comparant avec les inscriptions sur d'autres objets. Nous avons vu un grand nombre de boucliers contenant des vers persans, dont beaucoup sont ceux présentés ici, dans l'exposition du Museo Stibbert à Florence et à la Wallace Collection à Londres, mais il ne nous a pas été possible d'examiner ces boucliers «de près» et d'être ainsi en mesure de lire les inscriptions dans tous les cartouches ou bien d'observer les détails nécessaires pour un déchiffrement complet et correct.

\section{DATES}

Il est rare qu'on trouve une datation sur les boucliers. Dans notre «population» l'année ne se trouve indiquée que sur trois objets, mais sans qu'il soit précisé s'il s'agit de l'année de fabrication ou celle d'un événement.

Sur un bouclier ${ }^{80}$, dans un cartouche de la bordure qui contient la

80 Prague, Musée Naprstek, no. 19479. 
fin du do-beytī persan qui commence par «Le bouclier à la main de cet homme courageux...», nous y trouvons encore mentionné $r \cdot 1$, $i>$ gu ce qui signifie «daté $201 »$. Cette date, où manque sans doute le premier chiffre indiquant les milliers, doit être l'année 1201 de l'ère musulmane, ce qui correspond aux années 1786-87 de l'ère chrétienne. Et il est bien possible que le bouclier, d'après le caractère et l'exécution de son décor, ait été fabriqué vers la fin du XviIr ${ }^{\mathrm{c}}$ siècle.

Sur un autre bouclier qui porte une date ${ }^{81}$, nous lisons dans un lobe pointu du médaillon central

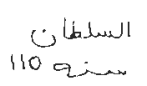

«le sultan, l'année 115»,

et dans un autre lobe pointu nous trouvons après quelques mots illisibles 110 / ain, «l'année 1151 ». Si nous supposons que le chiffre indiquant les milliers manque dans la première date et si nous ajoutons par conséquent le chiffre «1», nous obtenons l'année 1703-4 de l'ère chrétienne, tandis que l'autre date, «l'année 1151», correspond à l'année 1738-39 de l'ère chrétienne. Dans le texte de l'inscription qui est dans la bordure du même bouclier, est mentionné le nom du shāh CAbbās II (1642-1666) et aucune de ces dates ne correspond à l'époque de son règne. D'ailleures, d'après le caractère de son décor ce bouclier est beaucoup plus tardif ( $\mathrm{XIX}^{\mathrm{e}}$ siècle) et il est difficile de prendre toutes ces indications «historiques» au sérieux.

La dernière date que nous avons trouvée est placée dans un des quatre cartouches dans le champ d'un bouclier ${ }^{82}$. C'est l'inscription U U. W1, «131», qu'on pourrait supposer être l'année 1131 qui correspond à l'année 1718-19 de l'ère chrétienne. Zeller dans son catalogue indique l'année 1301 correspondant à l'année 1883-84 de l'ère chrétienne ${ }^{83}$. Il est bien possible que le point indiquant le zéro ait disparu, nous ne l'avons pas vu, car le bouclier lui-même pourrait être du $\mathrm{xIX}^{\mathrm{e}}$ siècle et l'inscription indiquant le nom du propriétaire pourrait être considérée comme authentique.

81 Paris, Musée de l'Armée, no. G 739 (Iran, x1x siècle). siècle).

82 Berne, Musée d'Histoire, no. 969 (Inde de l'Ouest, lère moitié du xrx

${ }_{83}$ Voir Rudolf ZELLER et Ernest F. RoHRER, Orientalische Sammlung Henri Moser-Cbarlottenfels; Beschreibender Kalalog der Waffensammlung, Berne, 1955, página 92. 


\section{NOM DU PROPRIÉTAIRE}

Ce n'est que sur un seul bouclier, celui dont nous venons de parler ${ }^{82}$, que nous trouvons le nom du propriétaire, qui est un certain Pāšã Beg, comme nous le dit le texte

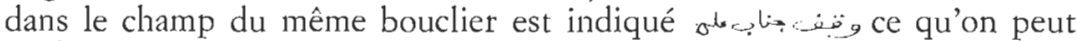
traduire soit comme «waqf de Genāb ${ }^{c} A l \bar{l}$ » ou «Ge nāb ${ }^{c} A l i ̄$ a constitué comme waqf». Il est impossible d'identifier les deux personnages, car leurs noms sont très communs.

\section{Signatures}

Nous n'avons trouvé le nom de l'artisan sur aucun bouclier, alors que c'est très fréquent par exemple sur les sabres ${ }^{84}$.

\section{TYPES D'ÉCRITURES}

Ce n'est pas notre but de faire ici une analyse paléographique approfondie, d'ailleurs on pourrait difficilement trouver une inscription qui présenterait un tel intérêt. Nous allons simplement présenter divers types d'écritures en relation avec le caractère du contenu des inscriptions.

Nous ne trouvons qu'un seul exemple d'écriture coufique ${ }^{85}$, ceci dans le cas d'une inscription mentionnant plusieurs fois le mot sultan avec divers titres, inscription qui est difficile, et inutile, à déchiffrer car elle est à la limite de la pseudo-épigraphie. D'ailleur le bouclier sur lequel elle se trouve ne peut être daté qu'au XIX ${ }^{\mathrm{e}}$ siècle et il ne s'agit que d'une assez mauvaise imitation de l'écriture coufique comme on l'a connue à son apogée.

84 Il semble que les noms des fabricants soient très rares sur les boucliers. Parmi le grand nombre de noms de fabricants d'autres armes, surtout des sabres, qui sont énumérés dans L. A. MAYER, Islamic Armourers and their Works, Genève, 1962, nous n'avons trouvé qu'un seul nom de fabricant, n'ayant signé qu'un seul bouclier. Sur la p. 56, nous lisons: «Muhammad, at an unspecified date, probably during the 10 th (16th) century, made a shield, now in the Oruzheynaya Palata, Moscow». Mais dans le livre Gosudarstvennaja oružejnaja palata moskovskogo Kremlja, Moscou, 1967, p. 124, cette signature a été lue Mumin-Muhamet Sa.

\&.5 Prague, Musée Naprstek, no. 4505 (Iran, $x 1 x^{\mathrm{c}}$ siècle). 
Plusieurs inscriptions à caractère religieux ${ }^{86}$, ainsi que les deux inscriptions exprimant des voeux ${ }^{87}$, sont exécutées en cursive ordinaire. On les trouve sur les boucliers qui peuvent être datés $\mathrm{du} \mathrm{XvI}^{\mathrm{e}}$ ou du XVII ${ }^{\mathfrak{C}}$ siècles et qui proviennent sans doute de la Turquie ottomane.

L'inscription à caractère religieux sur l'un de nos boucliers ${ }^{88}$ est en écriture cursive (tulut), mais c'est surtout la plupart des inscriptions mentionnant le nom d'un souverain ou bien seulement le mot sultan avec des titres ${ }^{i \prime}$ (iui sont en 1ulu. Les boucliers sur lesquels on les trouve peuvent être tous datés du $\mathrm{XIX}^{\mathrm{e}}$ siècle et ils ont été fabriqués sans doute en Iran.

Tous les morceaux de poésie persane sont en ta' ${ }^{\mathrm{C}}$ liq allant parfois jusqu'au nasta ${ }^{\mathrm{C}}$ lĩq, en ta ${ }^{\mathrm{C}}$ līq sont exécutées aussi les deux courtes inscriptions à caractère historique ${ }^{90}$, placées dans de petits médaillons alternant avec des cartouches dans lesquels se trouvent des vers persans exécutés eux aussi en taclīq.

\section{Pseudo-inscriptions}

Nous avons déjà constaté à propos de certains boucliers portant des inscriptions mentionnant le mot sultan avec des titres et même éventuellement le nom d'un souverain, que ces inscriptions n'ont pas de valeur historique mais plutôt un but décoratif. Ces inscriptions peuvent encore être déchiffrées, bien que souvent très difficilement, car le souci de l'artisan qui les a exécutées n'était pas de leur donner un sens précis mais plutôt de profiter de la valeur esthétique de l'écriture arabe en la considérant comme un élément décoratif. Nous avons dit que ces inscriptions étaient déjà à la limite de la pseudoépigraphie (Fig. 8). Mais nous trouvons sur huit autres boucliers des caractères ou groupes de caractères arabes qui n'ont aucun sens précis et que nous appelons alors des pseudo-inscriptions.

86 Vienne, Historisches Museum der Stadt Wien, no. 126.229 ('Turquie, 2ème moitié du xvil siècle); Paris, Musée de l'Armée, no. I 90 (Turquie, xvI ou xvil siècle); Vienne, Fleeresgeschichtliches Museum, l'exposition permanente (Turquie, xvit ${ }^{\mathrm{e}}$ siècle).

87 Vienne, Kunsthistorisches Museum, no. C 85 (Turquie, moitié du Xv1 siècle).

ss Berne, Musée d'Histoire, no. 1480 (Iran, xix siècle).

89 Prague, Musée Naprstek, no. 4505 (Iran, xIx ${ }^{\mathrm{e}}$ siècle); Prague, Musée Naprstek, no. 4453 (Iran, X1x siècle); Prague, Musée Naprstek, no. 19478 (Iran, xıx siècle); Prague, Musée Naprstek, no. 4506 (Iran, xix siècle); Prague, Musée Naprstek, no. 4507 (Iran, 2ème moitié du xix siècle); Paris, Musée de l'Armće, no. G 739 (Iran, xix ${ }^{*}$ siècle).

\%o Prague, Musée Napistek, no. 4495 (Iran, fin du Xvili ${ }^{\mathrm{c}}$ ou début du XIX siècle); Berne, Musée d'Histoire, no. Ps. 39 (Iran, XIx ${ }^{e}$ siècle). 

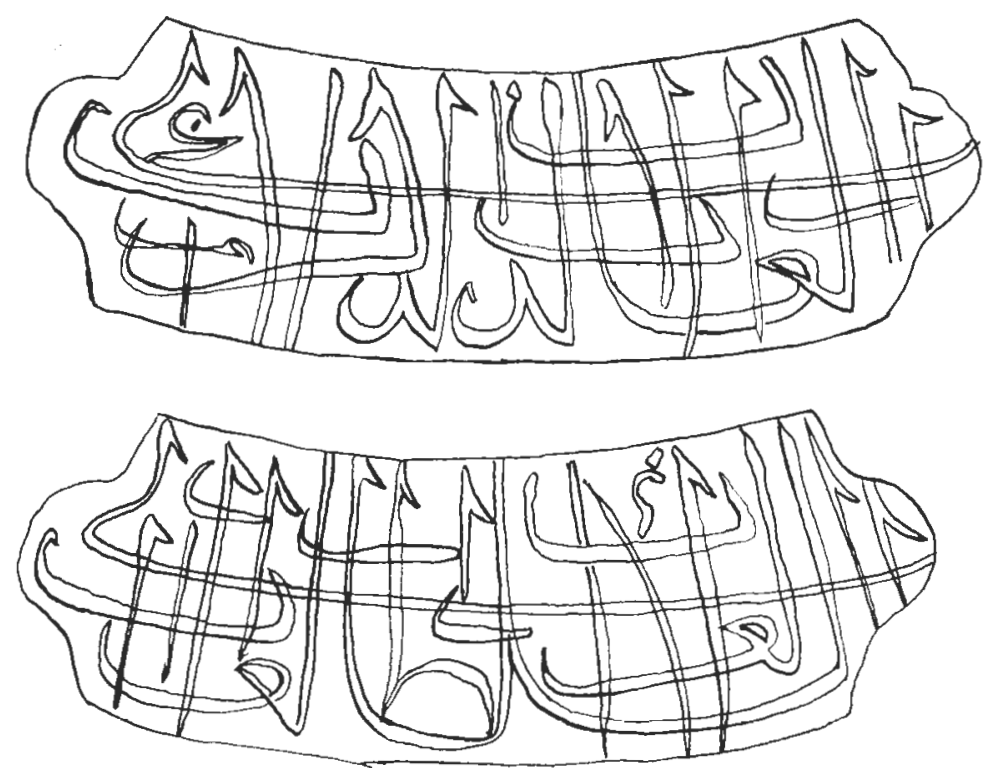

FIG. 8.-Exemples d'inscription à la limile de la pseudo-épigrapbie contenani certains titres de souverain (Prague, Musée Naprstek, no. 4507)

Un groupe de ces pseudo-inscriptions essaye apparement d'imiter certaines inscriptions contenant des vers persans exécutés en ta ${ }^{C}$ liq ${ }^{91}$. Ces pseudo-inscriptions sont toujours réparties dans huit cartouches allongés et huit médaillons qui alternent, ceci rappelant la répartition des inscriptions par exemple sur les boucliers où, dans la bordure, alternent les cartouches allongés et les cartouches circulaires, contenant les premiers des vers persans et les deuxièmes des titres de souverain ${ }^{92}$. Il ne semble pas qu'il s'agisse de la mauvaise imitation d'une véritable inscription par un artisan illetré, mais plutôt simplement de l'imitation de certains caractères ou groupes de caractères juxtaposés les uns à côté des autres sans aucune relation entre eux (Fig. 9).

91 Prague, Musée Naprstek, no. 16266 (Iran ou imitation européenne, 2ème moitié du XIX⿸厃㔾 ou début du $\mathrm{XX}^{\mathrm{e}}$ siècle); Berne, Musée d'Histoire, no. 1371 (Iran, 2ème moitié du xixe siècle); Paris, Musée de l'Armée, no. I 6999 (Iran, 2ème moitié du xrx siècle); Paris, Musée de l'Homme, no. 47.29.4 (Iran, Zème moitié du $\mathrm{XIX}^{\mathrm{e}}$ ou début $\mathrm{xx}$ e siècle); Paris, musée de l'Homme, no. 34.141 .2 (Iran, 2ème moitié du $\mathrm{XIX}^{\mathrm{C}}$ ou début du $\mathrm{xx}^{\mathrm{e}}$ siècle).

92 Prague, Musée Naprstek, no. 4495 (Iran, fin du xviII ${ }^{c}$ ou début du XIX ${ }^{\circ}$ siècle); Berne, Musée d'Histoire, no. Ps. 39 (Iran, XIxc siècle). 


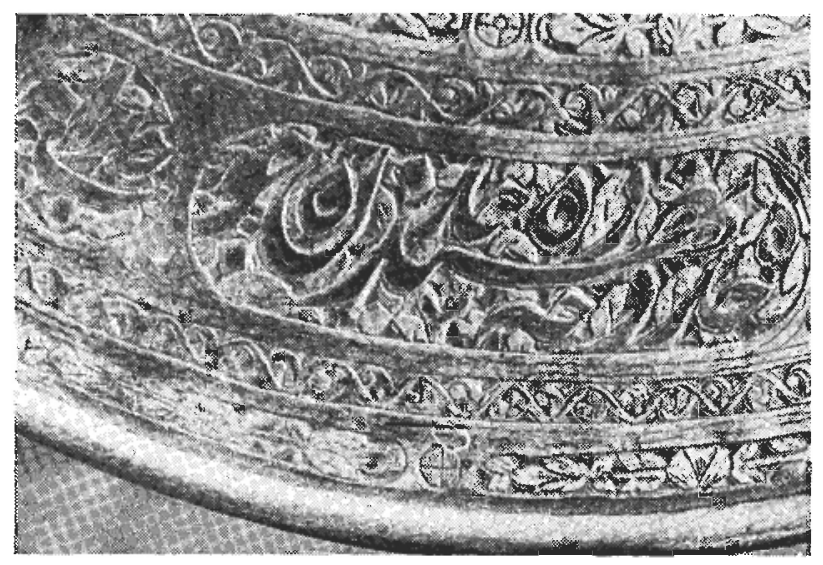

FIG. 9.--Iran, 2ème moilié du XIXe siècle (Berne, Musée d'Histoire, no. 1371) (Cliché: Kalus)

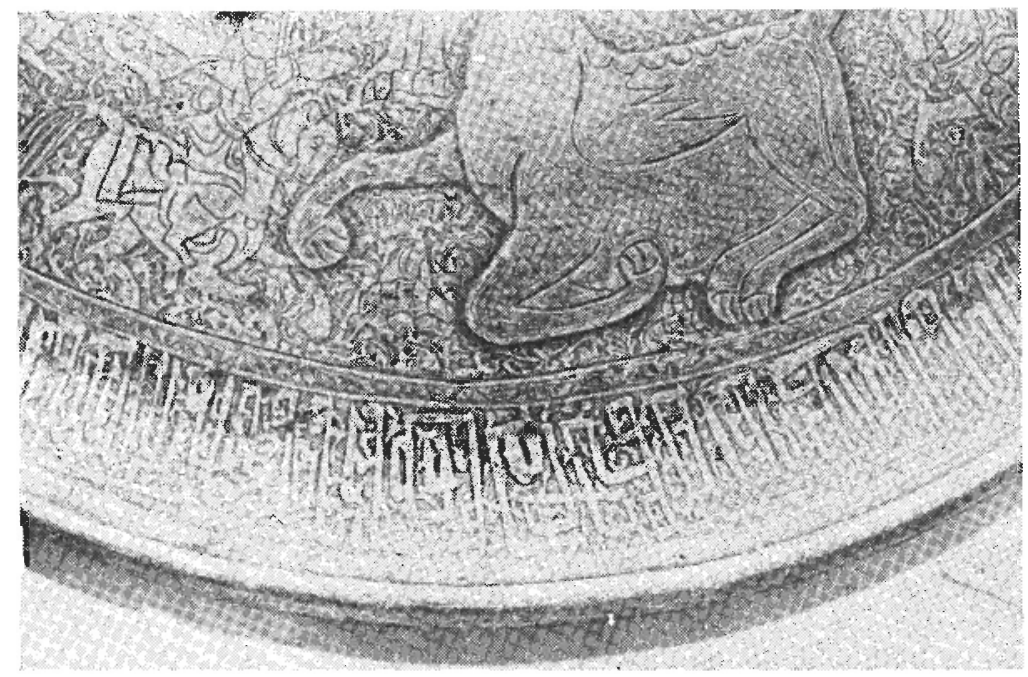

FIG. 10.-Iran, XIX' siècle (Paris, Musée de l'Armée, no. I 6670) (Cliché: Service Photographique du Musée de l'Armée) 
Une autre pseudo-inscription ${ }^{93}$ imite l'écriture coufique en remplissant sans intérruption une large bande formant la bordure. Ici rien ne permet de déterminer quel genre d'inscription on a cherché à imiter, peut-être un bandeau épigraphique monumental sur un monument architectural (Fig. 10).

Une autre pseudo-inscription est répartie dans le médaillon central ct dans les seize cartouches placés dans la bordure de l'un des boucliers ${ }^{94}$. Il s'agit ici aussis d'une imitation de l'écriture coufique. Notons que
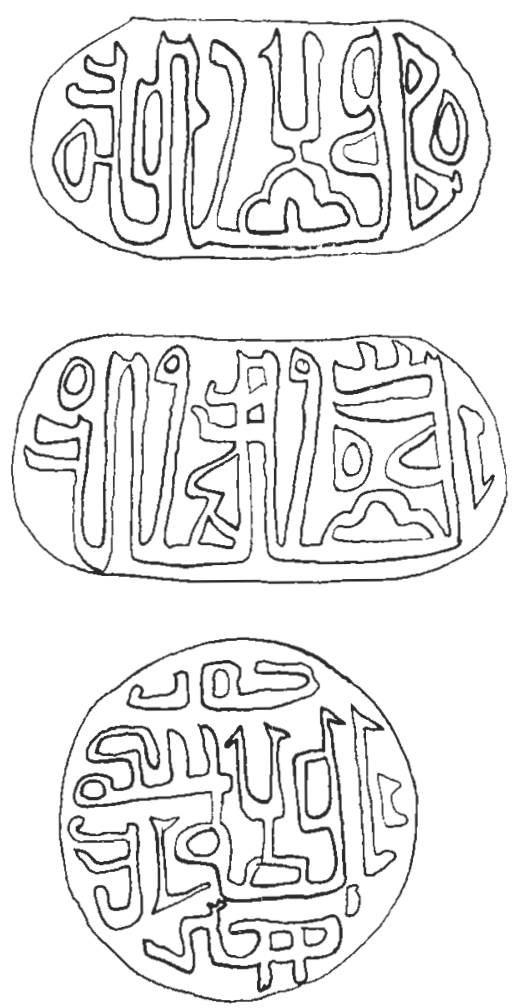

Fig. 11.-Exemple d'une pseudo-inscripiion (Prague, Musée Naprstek, no. 4504).

certaines de ces pseudo-inscriptions dans les cartouches de la bordure de ce bouclier donnent l'impression de vouloir évoquer en même temps l'écriture chinoise (Fig. 11).

${ }_{93}$ Paris, Musée de l'Armée, no. I 6670 (Iran, Xix $x^{e}$ siècle).

94 Prague, Musée Naptstek, no. 4504 (Iran, 2ème moitié du XIXe siècle). 
Notons encore une très mauvaise imitation des caractères arabes isolés dans des cartouches de la bordure d'un des boucliers étudiés ${ }^{95}$, dans d'autres cartouches de la même bordure on trouve aussi des caractères hindīs. Il s'agit d'une pièce «de curiosité» sans aucune valeur artistique.

Ainsi, comme on a pu le constater, les inscriptions sur les boucliers circulaires de l'Orient musulman présentées ci-dessus, sont d'une qualité inégale. A côté de «véritables» inscriptions, parmi lesquelles on peut compter la plupart des inscriptions à caractère religieux et les inscriptions exprimant des voeux, auxquelles peuvent s'ajouter un grand nombre d'inscriptions contenant des vers persans, on trouve également des inscriptions étant à la limite des pseudo-inscriptions ou étant tout simplement des pseudo-inscriptions. Ces deux dernières catégories se trouvent notamment sur les boucliers fabriqués au XIX $^{e}$ ou au début $\mathrm{du} \mathrm{xx}^{\mathrm{e}}$ siècle. Les inscriptions qui sont à la limite des pseudo-inscriptions sont à caractère «historique», ou plutôt pseudo-historique, contenant les titres d'un souverain, parfois même son nom, mais exécutées à une époque postérieure à celle du règne du souverain mentionné. Ces inscriptions sont plus ou moins bien réalisées et elles n'avaient d'autre but que décoratif, éventuellement elles devaient faire passer le bouclier comme étant celui du souverain mentionné, ou bien de son époque. Elles se rapprochent des pseudo-inscriptions qui sont typiques pour les objets «de curiosité», souvent de très mauvaise qualité.

Les inscriptions sont en rapport étroit avec le décor du bouclier ainsi qu'avec sa structure morphologique. Là où l'inscription est «authentique», le bouclier a la structure d'un bouclier destiné au combat et son décor reflète le décor qu'on trouve sur d'autres objets d'art mineurs caractéristiques de la même époque et du même milieu. Par contre, les boucliers ayant des inscriptions pseudo-historiques ou des pseudo-inscriptions ont une structure morphologique peu solide et le décor est souvent très hétéroclite, car le but était de fabriquer un objet «exotique» pour l'acheteur, objet qui ne devait jamais servir au combat. L'analyse des inscriptions peut donc contribuer d'une manière importante à la distinction des objets authentiques et des objets d'une valeur douteuse, ces derniers étant, hélas, souvent représentés dans de nombreuses collections privées ou publiques du monde occidental.

95 Berne, Musée d'Histoire, no, 1427 (Inde du Nord-Ouest, fin du XIx ${ }^{e}$ ou début du $\mathrm{Xx}^{\mathrm{c}}$ siècle, ou imitation européenne). 